\title{
From Hadronic Cross Section to the measurement of the Vacuum Polarization at KLOE: a fascinating endeavour
}

\author{
Graziano Venanzoni ${ }^{1, \star}$ \\ ${ }^{1}$ INFN Sezione di Pisa, Pisa, Italy
}

\begin{abstract}
The KLOE experiment at the $\phi$-factory DAФNE in Frascati is the first to have employed Initial State Radiation (ISR) to precisely determine the $e^{+} e^{-} \rightarrow \pi^{+} \pi^{-}(\gamma)$ cross section below $1 \mathrm{GeV}$. Such a measurement is particularly important to test the Standard Model (SM) calculation for the $(g-2)$ of the muon, where a long standing $3 \sigma$ discrepancy is observed. I will review the ISR activity in KLOE in the last 20 years from the measurement of the hadronic cross section to the first direct determination of the time-like complex running $\alpha(s)$ in the region below $1 \mathrm{GeV}$.
\end{abstract}

\section{Introduction: the Endurance expedition}

In August 1914, polar explorer Sir Ernest Shackleton boarded the Endurance and set sail for Antarctica, where he planned to cross the last uncharted continent on foot. In January 1915, after battling its way through a thousand miles of pack ice and only a day's sail short of its destination, the Endurance became locked in an island of ice (see Fig 1, left). Thus began the legendary ordeal of Shackleton and his crew of twenty-seven men. For ten months the ice-moored Endurance drifted northwest before it was finally crushed between two ice floes. With no options left, Shackleton and a 5 members crew left on 24 April 1916 on a 7 m boat, the James Caird, from Elephant Island in the South Shetland Islands for a near-impossible journey over 850 miles of the South Atlantic's heaviest seas to the island of South Georgia (see Fig. 1, right). Shackleton indeed succeeded to reach the island, and on 30 August, after four attempts, Shackleton was able to return to Elephant Island to rescue the party stranded there. Every single man survived.

With due proportion, this epic feat of endurance had many distinctive characters of the modern scientific research: experience the raw beauty and test the human spirit in an unknown (and often hostile) territory with perseverance and dedication; draw conclusions from observation, (incomplete) information, and inspiration; learn from errors. Such a mental attitude especially applies when the object of a research is a precision measurement, as was the trip of the James Caird boat to South Georgia, a tiny dot of land in a vast and hostile ocean.

\section{1999-2004: The first KLOE measurement of $\sigma\left(e^{+} e^{-} \rightarrow \pi^{+} \pi^{-}\right)$with ISR}

After more than twenty years from the first calculations [1], the late nineties saw a vigorous and renovated interest towards the use of Initial State Radiation process (ISR in the following) for the precise

^e-mail: graziano.venanzoni@lnf.infn.it 

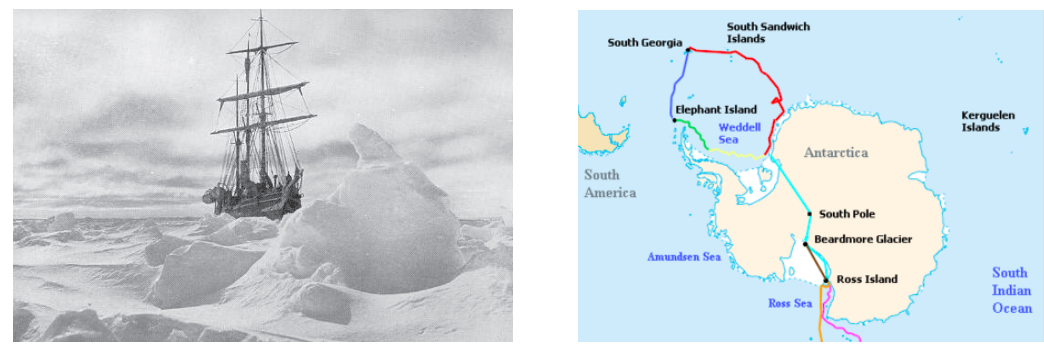

Figure 1. Left: The Endurance trapped in ice; -Photo by Frank Hurley, courtesy of the Macklin Collection; Right: Map of the sea routes of Endurance and James Caird: in red the voyage of Endurance; in yellow the drift in pack ice; in green the sea-ice drift after Endurance sinks; in blue the voyage of the boat James Caird.

measurement of the hadronic cross section [2]. The high luminosity reached at flavor factories made it possible to compensate the smaller cross section of the radiative process, making ISR an alternative and competitive method with respect to the traditional energy scan [3]. From the theoretical side the development of the PHOKHARA Monte Carlo event generator [4], based on exact QED NLO predictions, together with improved Monte Carlo codes for luminosity measurement, like BABAYAGA [5] or BHWIDE [6] and MCGPJ [7], made it possible to reach the sub per cent accuracy on the measurement of ISR cross sections [8].

From the experimental side ISR was first performed at KLOE [9, 10], to determine the cross section $e^{+} e^{-} \rightarrow \pi^{+} \pi^{-} \gamma$, soon followed by BaBar [11]. In the recent years this method was used to study a multitude of final states at BaBar [12], Belle [13, 14] and BES-III [15].

The first KLOE analysis of $e^{+} e^{-} \rightarrow \pi^{+} \pi^{-} \gamma$ cross section (called KLOE05 in the following) was performed during the years from 1999 to 2004 [16]. This analysis was mainly motivated by the discrepancy between the Standard Model prediction (SM in the following) and the measured value of the muon g-2 [17]. A large part of the uncertainty on the theoretical estimates comes from the leading order hadronic contribution $a_{\mu}^{\mathrm{HLO}}$, which at low energies is not calculable by perturbative QCD, but has to be evaluated with a dispersion integral using measured hadronic cross sections.

The process $e^{+} e^{-} \rightarrow \pi^{+} \pi^{-}$below $1 \mathrm{GeV}$ accounts for $\sim 70 \%$ of $a_{\mu}^{\mathrm{HLO}}$ [18], whose uncertainty dominates the SM evaluation of $a_{\mu}$.

At the time of KLOE05 there was a rather strong disagreement between $a_{\mu}^{\mathrm{HLO}}$ value obtained using data from $\tau$ decays into two- and four-pion final states after isospin-breaking corrections and $e^{+} e^{-}$based data. In particular while the SM prediction using $\tau$ data showed a difference less than $1 \sigma$ with the BNL experimental result, the $e^{+} e^{-}$based result disagreed by $\sim 3 \sigma$ [20] (see Fig. 2, left). In addition the $\pi^{+} \pi^{-}$spectral function from $e^{+} e^{-}$and isospin-corrected $\tau$ data were showing a systematic deviation in shape and normalisation [20].

KLOE05 and the following KLOE08 analyses used the so called Small Angle (SA) selection cuts: photons remaining undetected are restricted to a cone of $\theta_{\gamma}<15^{\circ}$ around the beamline (narrow cones in Fig. 2, right) and the two charged pion tracks are detected within $50^{\circ}<\theta_{\pi}<130^{\circ}$ (wide cones in Fig. 2, right). In this configuration, the direction of the photons is reconstructed from the tracks' momenta by closing kinematics: $\vec{p}_{\gamma} \simeq \vec{p}_{\text {miss }}=-\left(\vec{p}_{\pi^{+}}+\vec{p}_{\pi^{-}}\right)$. While these cuts guarantee a high statistics for ISR signal events and a reduced contamination from the resonant process $e^{+} e^{-} \rightarrow$ $\phi \rightarrow \pi^{+} \pi^{-} \pi^{0}$, in which the $\pi^{0}$ mimics the missing momentum of the photon(s), and from the final state radiation process $e^{+} e^{-} \rightarrow \pi^{+} \pi^{-} \gamma_{\mathrm{FSR}}$, a highly energetic photon emitted at small angle forces 

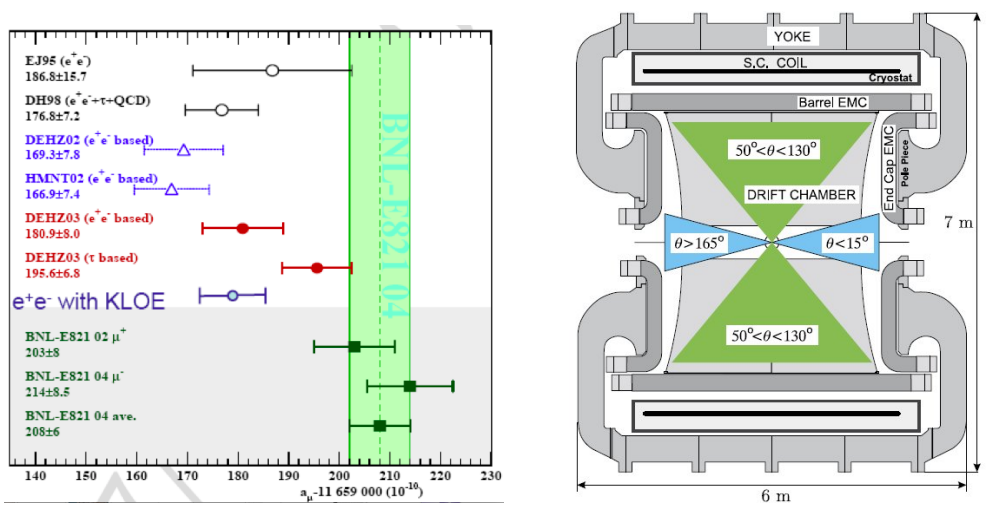

Figure 2. Left: Comparison between different theoretical predictions of the anomalous magnetic moment of the muon and the experimental measurement from E821 around 2004 when KLOE data were available. From F. Teubert presentation at ICHEP04 [19]. Right: Schematic view of the KLOE detector with selection regions.

the pions also to be at small angles (and thus outside the selection cuts), resulting in a kinematic suppression of events with $M_{\pi \pi}^{2}<0.35 \mathrm{GeV}^{2}$. The measurement of $\pi \pi \gamma$ cross section was normalized to the DAФNE luminosity using large angle Bhabha scattering with $0.3 \%$ total systematic uncertainty (using BABAYAGA), and the pion form factor was derived in the energy region between 0.35 and $0.95 \mathrm{GeV}^{2}$ by dividing the $\pi \pi \gamma$ cross section by the so called radiator function obtained with NLO PHOKHARA MC generator. Although with a limited accuracy of $1.3 \%$ equally divided between theory and experiment, the importance of KLOE05 was twofold:

- It established the use of ISR as a working technique to precisely measure the hadronic cross section with a competitive accuracy of the energy scan;

- It confirmed the $3 \sigma$ discrepancy of the muon g-2 between the direct measurement and the SM prediction based on $e^{+} e^{-}$data, as shown in Fig 2, left.

In addition KLOE05 allowed the KLOE Collaboration to get confidence into a novel technique which would have been refined in the following years.

\section{2005-2010: KLOE08 and KLOE10 analyses}

As most of the adventures in a new territory, the KLOE05 analysis was not free from errors. Particularly a bias in the evaluation of the trigger correction was found, which together with a reevaluation of the luminosity (due to an improved version of Bhabha generator BABAYAGA [5]), resulted in a few $\%$ discrepancy in the two spectra below the $\rho$ peak, and a slightly smaller value of $a_{\mu}^{\pi \pi}$ (below one standard deviation) [21]. In the meantime a new analysis called (KLOE08 in the following) was carried out with the same SA selection criteria for pion tracks and photon angles as of KLOE05 on $240 \mathrm{pb}^{-1}$ of data taken in 2002 [21]. With respect to KLOE05, KLOE08 profits from lower machine background and more stable DAФNE operation in 2002. Data filters and analysis technique were improved, together with a refined knowledge of the detector response and of the KLOE simulation and analysis, including a new version of generator PHOKHARA, with next-to-leading-order ISR and FSR corrections, as well as simultaneous emission of one ISR and one FSR photon [4]. 

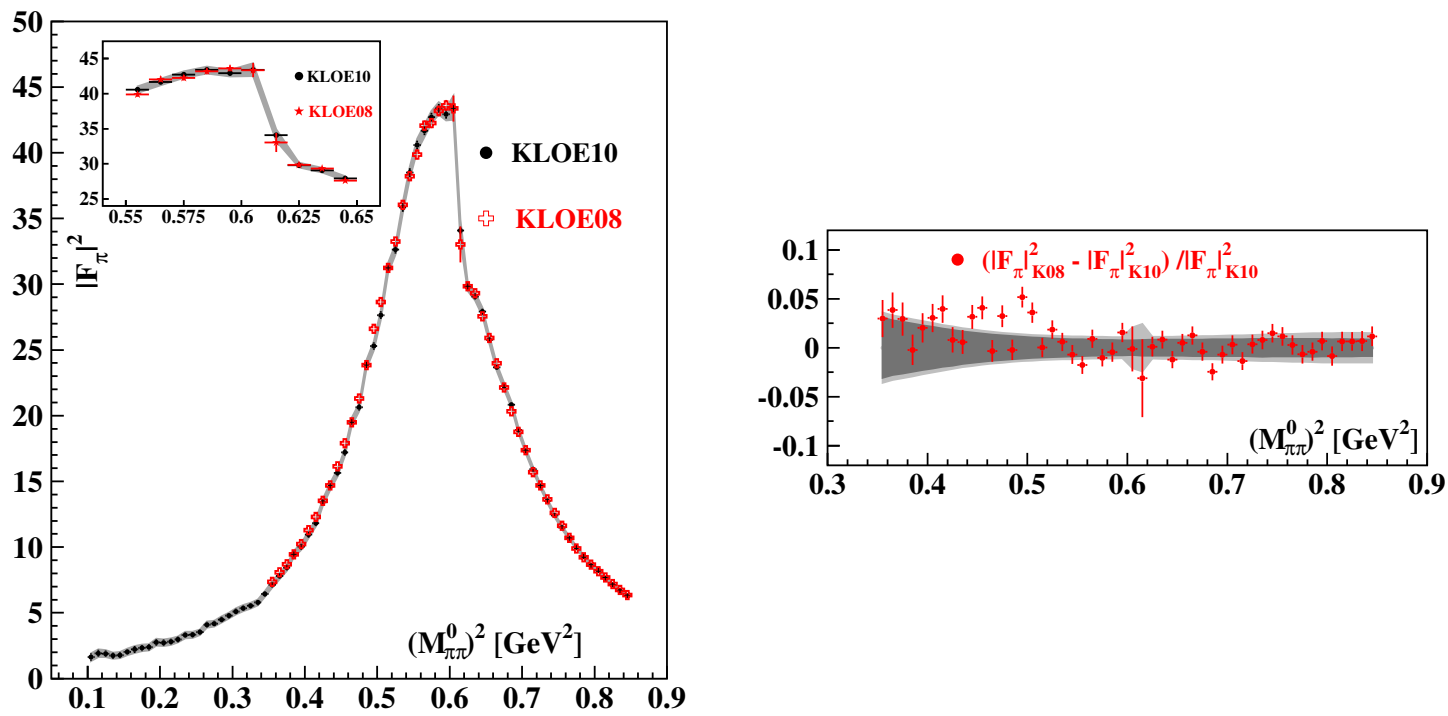

Figure 3. Comparison of KLOE10 with the previous KLOE result, KLOE08. Left: Pion form factor $\left|F_{\pi}\right|^{2}$. Right: Fractional difference between KLOE08 and KLOE10 results. The dark (light) gray band gives the statistical (total) error for the present result. Errors on KLOE08 points contain the combined statistical and systematic uncertainty.

All these refinement resulted in an improved $0.9 \%$ total systematic uncertainty consistent with KLOE05, with a total error smaller by $30 \%$, confirming the $3 \sigma$ discrepancy on the muon g- 2 . In view of the improvements of the analysis and the superior quality of the 2002 data, KLOE08 superseded KLOE05 [21].

Although the KLOE08 measurement had reached a remarkable $0.9 \%$ systematic error, the measured region was limited to $600 \mathrm{MeV}$, due to the SA selection requirement. To reach the dipion threshold, a new measurement (called KLOE10 in the following) was performed where a photon was detected in the calorimeter at large polar angles, $50^{\circ}<\theta_{\gamma}<130^{\circ}$, in the same region where also pion tracks are detected (so called Large Angle (LA) selection) [22]. However, compared to the measurements with photons at small angles, these conditions imply a reduction in statistics of about a factor of 5, and an increase of the background from the process $\phi \rightarrow \pi^{+} \pi^{-} \pi^{o}$, as well as the irreducible background from events with final state radiation and from $\phi$ radiative decays. To reduce the background contamination and the related uncertainties, the analysis was performed on $232.6 \mathrm{pb}^{-1}$ of data taken in 2006 at $1 \mathrm{GeV}$ (i.e. $20 \mathrm{MeV}$ below the $\phi$ mass). The KLOE10 analysis measured $a_{\mu}^{\pi \pi}$ in the region $0.1-0.85 \mathrm{GeV}^{2}$ with a $1 \%$ experimental systematic error and $0.9 \%$ theoretical one dominated by uncertainty on final state radiation (FSR) model. KLOE10 showed a good agreement with the previous KLOE measurements (see Fig. 3), allowing to cover $70 \%$ of the leading order hadronic contribution to the muon anomaly with $1 \%$ total error. It showed also a reasonable agreement with the results from the Novosibirsk experiments CMD-2 and SND (see Fig. 4, left); while comparing with the BaBar result it showed agreement below $0.4 \mathrm{GeV}^{2}$ (corresponding to a value of $M_{\pi \pi}$ of $630 \mathrm{MeV}$ ), while above $0.4 \mathrm{GeV}^{2}$ the $\mathrm{BaBar}$ result is higher by $2-3 \%$ (Fig. 4, right).

The KLOE10 data allowed also to test the validity of different models for the $\pi^{+} \pi^{-}$final state photon emission in the forward-backward asymmetry, showing good agreement with the point-like pions (so called scalar QED) assumption (see Fig. 5) [8, 23-25]. 

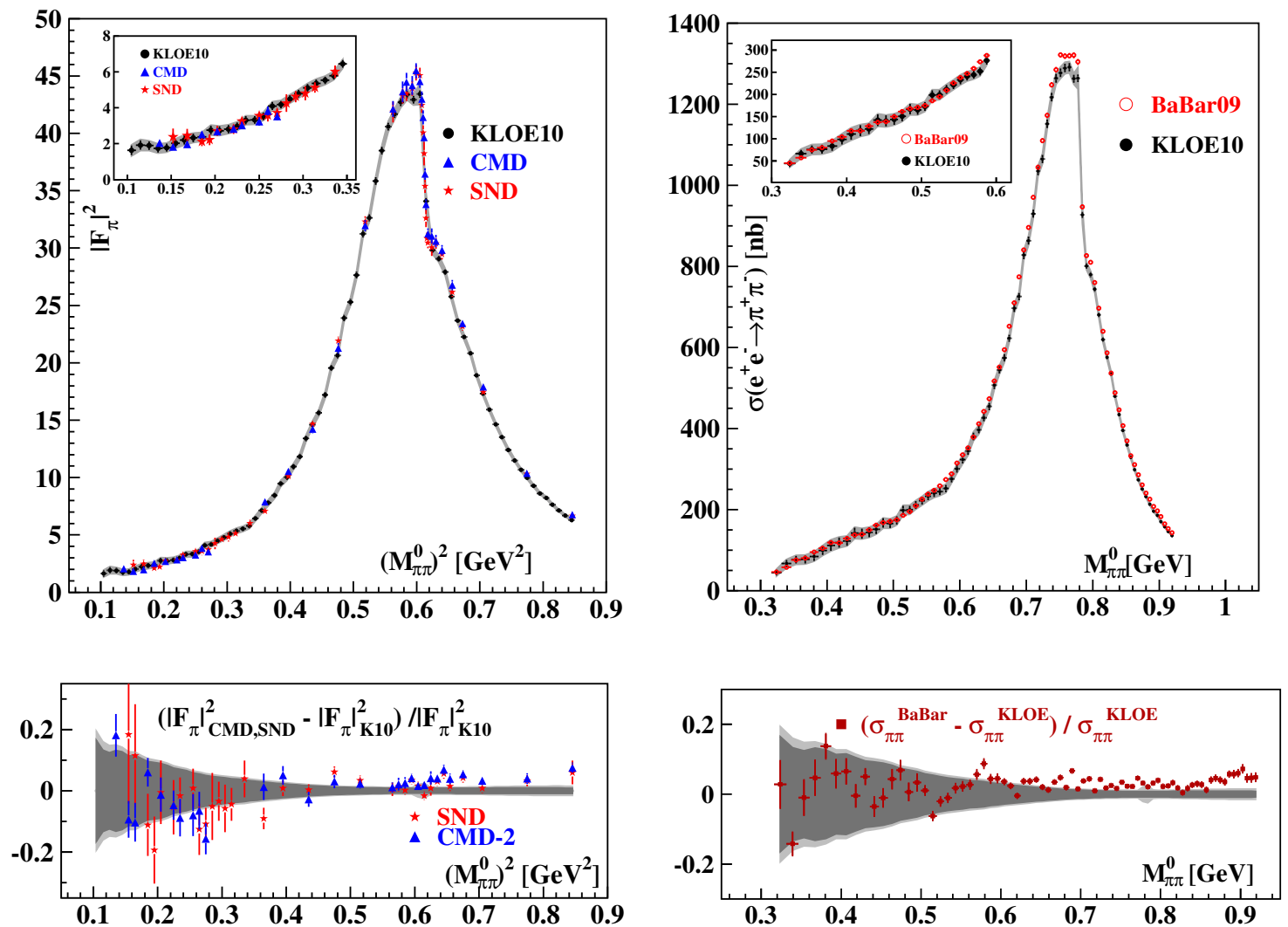

Figure 4. Top left: $\left|F_{\pi}\right|^{2}$ from CMD-2 [26, 27], SND [28] and the present KLOE result as function of $\left(M_{\pi \pi}^{0}\right)^{2}$. Bottom left: Fractional difference between CMD-2 or SND and KLOE. Top right: $\sigma_{\pi \pi}^{\text {bare }}$ from BaBar [29] and the new KLOE result as function of $M_{\pi \pi}^{0}$. Bottom right: Fractional difference between BaBar and KLOE. CMD-2, SND and BaBar data points have the total uncertainty attached. The dark (light) band in the lower plots shows statistical (total) error of the KLOE result.

\section{2010-2012: KLOE12 analysis}

While KLOE08 and KLOE10 were independent measurements with respect to the data set and angular selection, they were both normalized to the DAФNE luminosity, and used the radiation function to obtain the pion form factor and $a_{\mu}$. As shown in Ref. [29] it's possible to extract the pion form factor by normalizing to the muon ISR differential cross section. In this approach the integrated luminosity as well as the radiation function $\mathrm{H}$ cancel in the ratio, as does the vacuum polarisation. In addition using the same fiducial volume, acceptance corrections to $\pi \pi \gamma$ and $\mu \mu \gamma$ spectra almost cancel resulting in a per mille systematic uncertainty. However the price to pay is to perform an additional analysis on muon events at subpercent level. The same sample of $239.2 \mathrm{pb}^{-1}$ of KLOE08 was analyzed with the small angle photon selection (called KLOE12 in the following) [30]. While the analysis for $\pi \pi \gamma$ is essentially the same as for KLOE08, the analysis for $\mu \mu \gamma$ is new and is based on the following main features: 1) separation between $\mu \mu \gamma$ and $\pi \pi \gamma$ events obtained assuming the final state of two charged particles with equal track mass $M_{T R K}$ and one photon: the $M_{T R K}<115 \mathrm{MeV}\left(M_{T R K}>130 \mathrm{MeV}\right)$ selection leads to $9 \times 10^{5}\left(3.1 \times 10^{6}\right)$ candidate $\mu \mu \gamma(\pi \pi \gamma)$ events, this selection is checked against other 

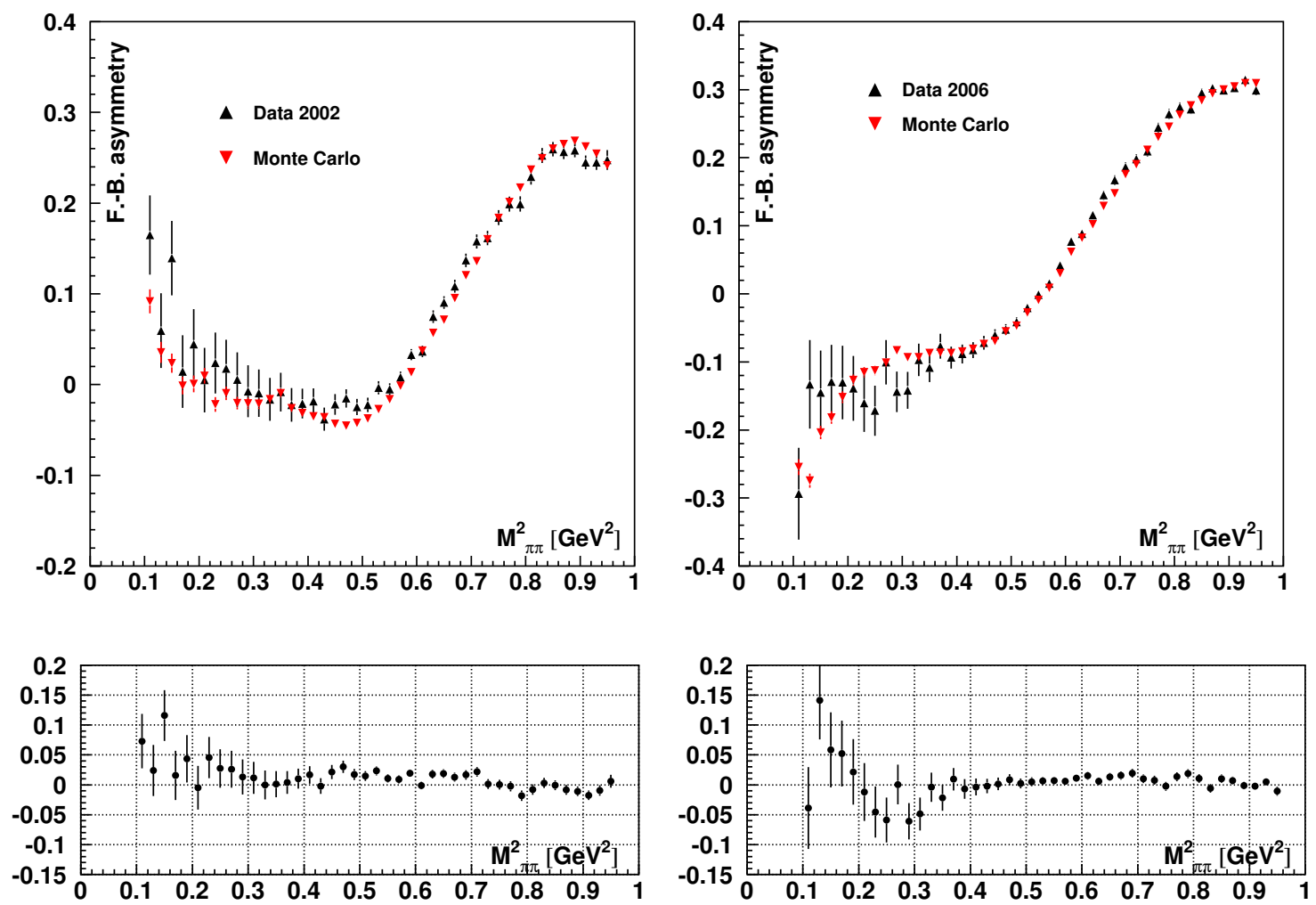

Figure 5. Top left: Forward-Backward asymmetry for data taken at $\sqrt{s}=M_{\phi}$ in 2002, and the corresponding

Monte Carlo prediction using the PHOKHARA v6.1 generator. Bottom left: Absolute difference between the asymmetries from data and Monte Carlo prediction. Top right: Forward-Backward asymmetry for data taken at $\sqrt{s} \simeq 1000 \mathrm{MeV}$ in 2006, and the corresponding Monte Carlo prediction using the PHOKHARA v6.1 generator. Bottom right: Absolute difference between the asymmetries from data and Monte Carlo prediction. From Ref. [8]

techniques, such as a kinematic fit or tighter cuts on the quality of the charged tracks, all providing consistent results; 2) trigger, particle identification and tracking efficiencies checked from data control samples. The $\mu \mu \gamma$ cross section measurement was compared with the one obtained by PHOKHARA MC [4], and a good agreement is found [30]. Then the pion form factor has been extracted and compared with the one from KLOE10, showing good agreement (see Fig. 6, left). The value for $a_{\mu}^{\pi \pi}$ computed with KLOE12 was in good agreement with the previous KLOE results, providing therefore a strong cross check of the systematics and confirming the $3 \sigma$ discrepancy between the experimental value and the Standard Model prediction of the muon g-2 (see Fig. 6 right).

\section{2013-2016: Measurement of the running of the fine structure constant below $1 \mathrm{GeV}$}

With about $2 \mathrm{fb}^{-1}$ of luminosity collected in 2004-2005, the KLOE-2 Collaboration - the successor of KLOE - embarked in a full statistics analysis of the $\mu \mu \gamma$ cross section with a twofold motivation:

1. to search for a light vector boson [38]; 

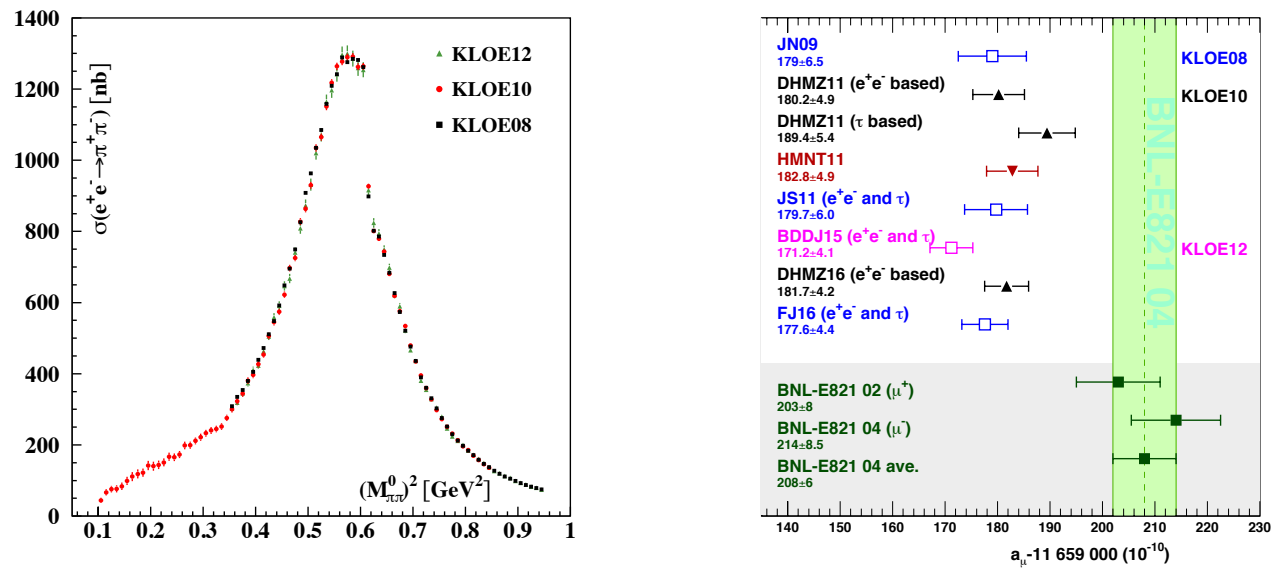

Figure 6. Left: pion form factor $\left|F_{\pi}\right|^{2}$ obtained by $\pi \pi \gamma / \mu \mu \gamma$ ratio (KLOE12) compared with previous KLOE measurements. Right: compilation of recent results for $a_{\mu}$ compared with the experimental value. The SM predictions refer to: JN09 [31], DHMZ11 [32], HLMNT11 [33], JS11 [34], BDDJ15 [35], DHMZ16 [36], FJ16 [37]. KLOE08 [21], KLOE10 [22], and KLOE12 [30] refer to the compilations where KLOE data are included.

2. to measure the running of the $Q E D$ coupling constant $\alpha(s)$ below $1 \mathrm{GeV}$.

The analysis of the so called $u$-boson is in progress. In the following we will discuss the measurement of the running of the fine structure constant $\alpha$ in the time-like region $0.6<\sqrt{s}<0.975 \mathrm{GeV}$ [39].

The strength of the coupling constant is measured as a function of the momentum transfer of the exchanged photon $\sqrt{s}=M_{\mu \mu}$ where $M_{\mu \mu}$ is the $\mu^{+} \mu^{-}$invariant mass. The value of $\alpha(s)$ is extracted from the ratio of the differential cross section for the process $e^{+} e^{-} \rightarrow \mu^{+} \mu^{-} \gamma(\gamma)$ with the photon emitted in the Initial State (ISR) to the corresponding cross section obtained from Monte Carlo (MC) simulation with the coupling set to the constant value $\alpha(s)=\alpha(0)$ :

$$
\left|\frac{\alpha(s)}{\alpha(0)}\right|^{2}=\frac{\left.d \sigma_{\text {data }}\left(e^{+} e^{-} \rightarrow \mu^{+} \mu^{-} \gamma(\gamma)\right)\right|_{I S R} / d \sqrt{s}}{\left.d \sigma_{M C}^{0}\left(e^{+} e^{-} \rightarrow \mu^{+} \mu^{-} \gamma(\gamma)\right)\right|_{I S R} / d \sqrt{s}}
$$

To obtain the ISR cross section, the observed cross section must be corrected for events with one or more photons in the final state (FSR). This has been done by using the PHOKHARA MC event generator, which includes next-to-leading-order ISR and FSR contributions [4]. Figure 7, left, shows the ratio of the $\mu^{+} \mu^{-} \gamma$ cross-section from data with the corresponding NLO QED calculation from PHOKHARA generator including the Vacuum Polarization effects.

The agreement between the two cross sections is excellent.

We use Eq. (1) to extract the running of the effective QED coupling constant $\alpha(s)$. By setting in the $\mathrm{MC}$ the electromagnetic coupling to the constant value $\alpha(s)=\alpha(0)$, the hadronic contribution to the photon propagator, with its characteristic $\rho-\omega$ interference structure, is clearly visible, see Fig. 7, right. The prediction from Ref.[40] is also shown. While the leptonic part is obtained by perturbation theory, the hadronic contribution to $\alpha(s)$ is obtained via an evaluation in terms of a weighted average 

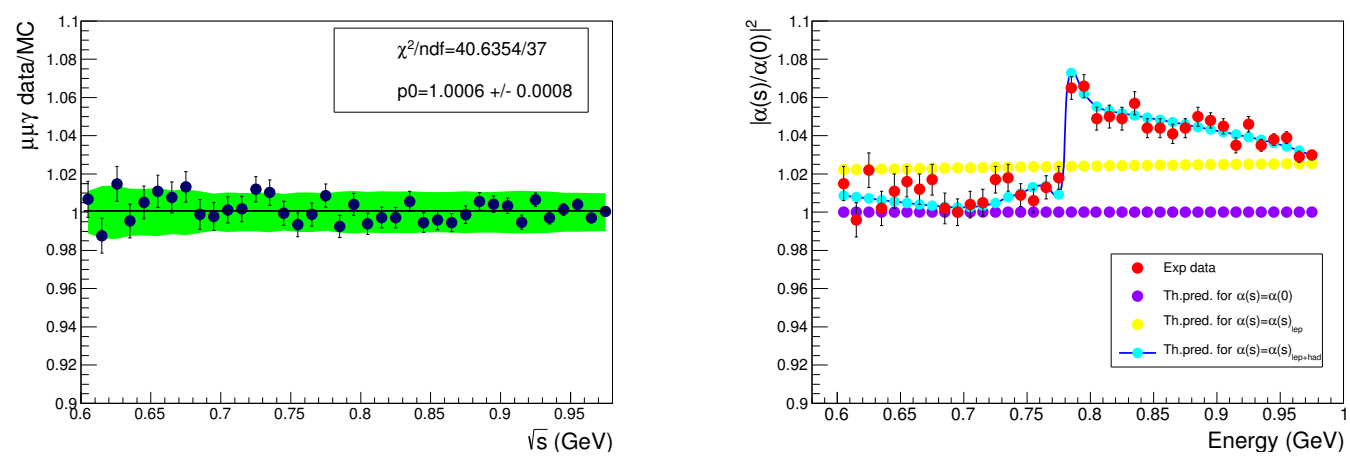

Figure 7. Left: Ratio between the measured differential $\mu^{+} \mu^{-} \gamma$ cross section and the MC prediction from PHOKHARA. The green band shows the systematic error. Right: The square of the modulus of the running $\alpha(s)$ in units of $\alpha(0)$ compared with the prediction (provided by the alphaQED package [40]) as a function of the dimuon invariant mass. The red points are the KLOE data with statistical errors; the violet points are the theoretical prediction for a fixed coupling $(\alpha(s)=\alpha(0))$; the yellow points are the prediction with only virtual lepton pairs contributing to the shift $\Delta \alpha(s)=\Delta \alpha(s)_{\text {lep }}$, and finally the points with the solid line are the full QED prediction with both lepton and quark pairs contributing to the shift $\Delta \alpha(s)=\Delta \alpha(s)_{\text {lep }+ \text { had }}$.

compilation of $R_{\text {had }}(s)$, based on the available experimental $e^{+} e^{-} \rightarrow$ hadrons annihilation data (for an up to date compilation see [41] and references therein).

For comparison, the prediction with constant coupling (no running) and with only lepton pairs contributing to the running of $\alpha(s)$ is given.

By including statistical and systematics errors, we exclude the only-leptonic hypothesis at $6 \sigma$ which is the strongest direct evidence ever achieved by a collider experiment ${ }^{1}$.

\subsection{Extraction of Real and Imaginary part of $\Delta \alpha(s)$}

By using the definition of the running of $\alpha$ the real part of the shift $\Delta \alpha(s)$ can be expressed in terms of its imaginary part and $|\alpha(s) / \alpha(0)|^{2}$ :

$$
\operatorname{Re} \Delta \alpha=1-\sqrt{|\alpha(0) / \alpha(s)|^{2}-(\operatorname{Im} \Delta \alpha)^{2}} .
$$

The imaginary part of $\Delta \alpha(s)$ can be related to the total cross section $\sigma\left(e^{+} e^{-} \rightarrow \gamma^{*} \rightarrow\right.$ anything $)$, where the precise relation reads [18, 31, 45]: $\operatorname{Im} \Delta \alpha=-\frac{\alpha}{3} R(s)$, with $R(s)=\sigma_{\text {tot }} / \frac{4 \pi|\alpha(s)|^{2}}{3 s} . R(s)$ takes

into account leptonic and hadronic contribution $R(s)=R_{l e p}(s)+R_{\text {had }}(s)$, where the leptonic part corresponds to the production of a lepton pair at lowest order taking into account mass effects:

$$
R_{\text {lep }}(s)=\sqrt{1-\frac{4 m_{l}^{2}}{s}}\left(1+\frac{2 m_{l}^{2}}{s}\right), \quad(l=e, \mu, \tau) .
$$

\footnotetext{
${ }^{1}$ The first evidence for the hadronic VP came from the ACO experiment which found an evidence at $3 \sigma$ of the $\phi$ contribution to the process $e^{+} e^{-} \rightarrow \mu^{+} \mu^{-}$in the region $\pm 6 \mathrm{MeV}$ around the $\phi$ peak (at 1019.4 MeV) [42]. The strongest evidence for hadronic VP effect comes from the muon g-2 experiment; at CERN it was determined at more than $7 \sigma$ level $[43,44]$.
} 
In the energy region around the $\rho$-meson we can approximate the hadronic cross section by the $2 \pi$ dominant contribution:

$$
R_{\text {had }}(s)=\frac{1}{4}\left(1-\frac{4 m_{\pi}^{2}}{s}\right)^{\frac{3}{2}}\left|F_{\pi}^{0}(s)\right|^{2},
$$

where $F_{\pi}^{0}$ is the pion form factor deconvolved: $\left|F_{\pi}^{0}(s)\right|^{2}=\left|F_{\pi}(s)\right|^{2}\left|\frac{\alpha(0)}{\alpha(s)}\right|^{2}$.

The results obtained for the $2 \pi$ contribution to the imaginary part of $\Delta \alpha(s)$ by using the KLOE pion form factor measurement[30], are shown in Fig. 8 and compared with the values given by the $R_{\text {had }}(s)$ compilation of Ref. [40] using only the $2 \pi$ channel, with the KLOE data removed (to avoid correlations).
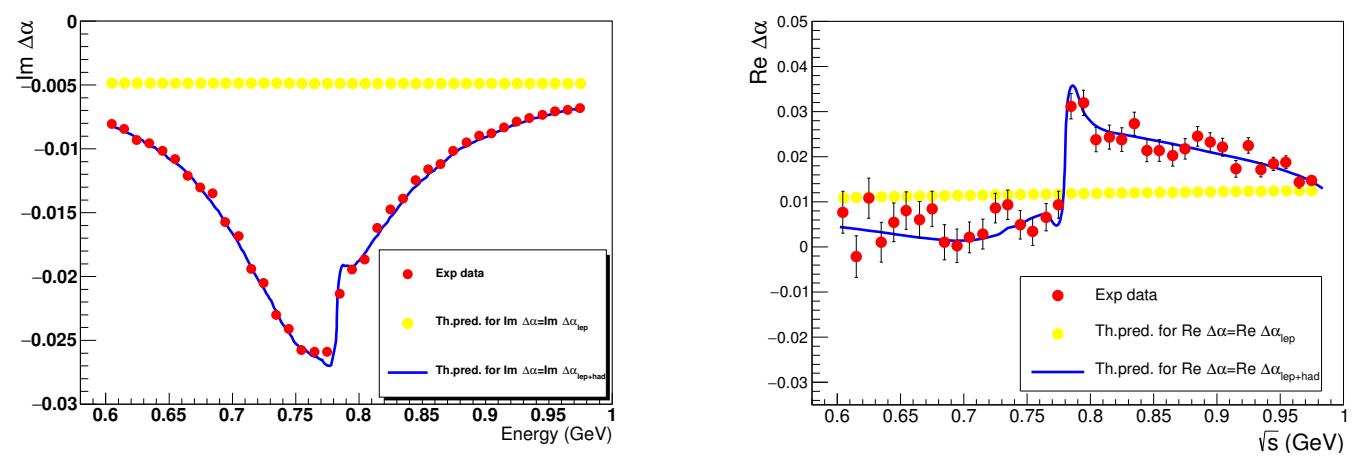

Figure 8. Left: $\operatorname{Im} \Delta \alpha$ extracted from the KLOE data compared with the values provided by alphaQED routine (without the KLOE data) for $\operatorname{Im} \Delta \alpha=\operatorname{Im} \Delta \alpha_{\text {lep }}$ (yellow points) and $\operatorname{Im} \Delta \alpha=\operatorname{Im} \Delta \alpha_{\text {lep+had }}$ only for $\pi \pi$ channels (blue solid line). Right: $\operatorname{Re} \Delta \alpha$ extracted from the experimental data with only the statistical error included compared with the alphaQED prediction (without the KLOE data) when $\operatorname{Re} \Delta \alpha=\operatorname{Re} \Delta \alpha_{\text {lep }}$ (yellow points) and $\operatorname{Re} \Delta \alpha=\operatorname{Re} \Delta \alpha_{\text {lep+had }}$ (blue solid line).

The extraction of the $\operatorname{Re} \Delta \alpha$ has been performed using the Eq. (2) and it is shown in Fig. 8, right. The experimental data with only the statistical error included have been compared with the alphaQED prediction when $\operatorname{Re} \Delta \alpha=\operatorname{Re} \Delta \alpha_{\text {lep }}$ (yellow points in the colour Figure) and $\operatorname{Re} \Delta \alpha=\operatorname{Re} \Delta \alpha_{\text {lep+had }}$ (dots with solid line). As can be seen, an excellent agreement for $\operatorname{Re} \Delta \alpha(\mathrm{s})$ has been obtained with the data-based compilation. 
Finally $\operatorname{Re} \Delta \alpha$ has been fitted by a sum of the leptonic and hadronic contributions, where the hadronic contribution is parametrized as a sum of the $\rho(770), \omega(782)$ and $\phi(1020)$ resonance components and a non-resonant term. The product of the branching fractions has been extracted:

$$
B R\left(\omega \rightarrow \mu^{+} \mu^{-}\right) B R\left(\omega \rightarrow e^{+} e^{-}\right)=\left(4.3 \pm 1.8_{\text {stat }} \pm 2.2_{\text {syst }}\right) \cdot 10^{-9},
$$

where the first error is statistical and the second systematic. By multiplying by the phase space factor $\xi=\left(1+2 \frac{m_{\mu}^{2}}{m_{\omega}^{2}}\right)\left(1-4 \frac{m_{\mu}^{2}}{m_{\omega}^{2}}\right)^{1 / 2}$ and assuming lepton universality, $B R\left(\omega \rightarrow \mu^{+} \mu^{-}\right)$can be extracted:

$$
B R\left(\omega \rightarrow \mu^{+} \mu^{-}\right)=\left(6.6 \pm 1.4_{\text {stat }} \pm 1.7_{\text {syst }}\right) \cdot 10^{-5}
$$

compared to $B R\left(\omega \rightarrow \mu^{+} \mu^{-}\right)=(9.0 \pm 3.1) \cdot 10^{-5}$ from PDG [46].

\section{Conclusions and Outlook}

The last 20 years have seen a flourishing use of the ISR to measure the hadronic cross sections at flavor factories. Being more than a poor man's alternative to scan energies the ISR represents an alternate, independent and complementary way to determine those cross sections with different systematic errors. Such a success story was possible thanks to dedicated work both from the theoreticians developing Monte Carlo generators like PHOKHARA and BABAYAGA@ NLO which were fundamental to achieve sub percent precision, as well as from experimental groups using ISR not only to determine hadronic cross sections but also for spectroscopy studies. In that respect the KLOE measurements of hadronic cross section was a significant contribution to this field ${ }^{2}$ :

- It proved the ISR as a working tool to precisely measure the hadronic cross sections;

- It confirmed the $3 \sigma$ discrepancy between the Standard Model prediction for the g-2 and the measured value, and the disagreement with the SM prediction based on $\tau$ data. These two sets of data are now in agreement with each other, once the vector meson and $\rho-\gamma$ mixing effects are properly accounted for [47];

- It allowed a first direct measurement of the time-like complex running $\alpha(s)$. That procedure would be helpful to solve a problem in the treatment of the narrow resonances to $a_{\mu}$ and to the running of $\alpha[41]$;

- By the close contact and interactions with theorists it contributed to the inter-comparisons and refinements of the Monte Carlo codes [8].

Thanks to the worldwide (experimental and theoretical) efforts the accuracy of leading-order hadronic vacuum polarization contribution, $a_{\mu}^{\mathrm{HLO}}$, has improved to $\sim 4 \times 10^{-10}$. Although corresponding to a remarkable fractional uncertainty of $0.6 \%$, it still constitutes the main uncertainty of the SM prediction to $a_{\mu}$. New experiments at Fermilab and J-PARC aiming at a fourfold improved precision are underway $[48,49]$. Together with a fourfold improved precision on the experimental side, an improvement of the LO hadronic contribution is highly desirable. An interesting possibility in this respect is a novel approach aiming to determine $a_{\mu}^{\mathrm{HLO}}$ from a direct space-like measurement of $\alpha(t)$ as proposed in Refs. [50, 51] (see also [52]) recently.

\footnotetext{
${ }^{2}$ As underlined also by more than 600 citations of the corresponding articles $[16,21,22,30]$.
} 


\section{Acknoweldgements}

The ISR measurement at KLOE was first of all a story of passion, friendship, committement and dedication. I would like to express my gratitude to the members of the KLOE/KLOE-2 Collaboration who made this possible and with whom I had the privilige to collaborate, particularly: P. Beltrame, F. Curciarello, V. De Leo, A. Denig, S. Di Falco, M. Incagli, W. Kluge, J. Lee-Franzini, P. Lukin, G. Mandaglio, S. Müller, F. Nguyen, A. Palladino, and B. Valeriani. Many friends and colleagues gave useful suggestions, help, criticism and support during the development of the analyses: A. Arbuzov, M. Benayoun, C.M. Carloni Calame, H. Czyż, S. Eidelman, G. Fedotovich, F. Ignatov, F. Jegerlehner, A. Keshavarzi, J.H. Kühn, G. Montagna, O. Nicrosini, L. Pancheri, M. Passera, F. Piccinini, G. Rodrigo, O. Shekhtostova, T. Teubner, and L. Trentadue. To all of them, and to the whole Radio Monte Carlo Working Group, goes my sincere gratitude and appreciation. Finally I would like to thank Rita Bertelli for her precious work and assistance during all these years.

\section{References}

[1] V. N. Baier, V. A. Khoze, ZHETF 48(1965) 946, Yad. Fiz. 2 (1965) 287; V. N. Baier, V. A. Khoze, Sov. Phys. JETP 21 (1965) 629, 1145

[Zh. Eksp. Theor. Fiz. 48 (1965) 946; V. N. Baier and V. S. Fadin Phys. Lett. 27B (1968) 223; G. Pancheri, Nuovo Cim. A 60 (1969) 321; M. Greco, G. Pancheri, Y. N. Srivastava, Nucl. Phys. B 101 (1975) 234; M. S. Chen, P. M. Zerwas,

Phys. Rev. D 11 (1975) 58.

[2] A. B. Arbuzov et al., JHEP 9710:006, 1997; A. B. Arbuzov, E. A. Kuraev, N. P. Merenkov, L. Trentadue, JHEP 9812:009, 1998; M. Benayoun, S. I. Eidelman, V. N. Ivanchenko, Z. K. Silagadze, Mod. Phys. Lett. A 14 (1999) 2605; S. Binner, J. H. Kühn, K. Melnikov, Phys. Lett. B 459 (1999) 279.

[3] See for example W. Kluge, Nucl. Phys. Proc. Suppl. 181-182 (2008) 280; V. P. Druzhinin, S. I. Eidelman, S. I. Serednyakov and E. P. Solodov, Rev. Mod. Phys. 83 (2011) 1545.

[4] F. Campanario, H. Czyż, J. Gluza, M. Gunia, T. Riemann, G. Rodrigo and V. Yundin, JHEP 1402 (2014) 114; H. Czyż, M. Gunia and J. H. Kühn, JHEP 1308 (2013) 110; H. Czyż, A. Grzelinska, J. H. Kühn and G. Rodrigo, Eur. Phys. J. C 39 (2005) 411; H. Czyż, A. Grzelinska, J. H. Kühn and G. Rodrigo, Eur. Phys. J. C 33 (2004) 333; H. Czyż, A. Grzelinska, J. H. Kühn and G. Rodrigo, Eur. Phys. J. C 27 (2003) 563; J. H. Kühn and G. Rodrigo, Eur. Phys. J. C 25 (2002) 215; G. Rodrigo, H. Czyż, J. H. Kühn and M. Szopa, Eur. Phys. J. C 24 (2002) 71; G. Rodrigo, A. Gehrmann-De Ridder, M. Guilleaume and J. H. Kühn, Eur. Phys. J. C 22 (2001) 81.

[5] G. Balossini, C. Bignamini, C. M. C. Calame, G. Montagna, O. Nicrosini and F. Piccinini, Phys. Lett. B 663 (2008) 209; G. Balossini, C. M. Carloni Calame, G. Montagna, O. Nicrosini and F. Piccinini, Nucl. Phys. B 758 (2006) 227; C. M. Carloni Calame, Phys. Lett. B 520 (2001) 16; C. M. Carloni Calame, C. Lunardini, G. Montagna, O. Nicrosini and F. Piccinini, Nucl. Phys. B 584 (2000) 459.

[6] S. Jadach, W. Placzek and B. F. L. Ward, Phys. Lett. B 390 (1997) 298.

[7] A. B. Arbuzov, G. V. Fedotovich, F. V. Ignatov, E. A. Kuraev and A. L. Sibidanov, Eur. Phys. J. C 46 (2006) 689.

[8] S. Actis et al., Eur. Phys. J. C 66 (2010) 585.

[9] S. Spagnolo, Eur. Phys. J. C 6 (1999) 637.

[10] G. Cataldi, A. Denig, W. Kluge, G. Venanzoni, KLOE memo 195 (Aug. 1999);

Frascati Physics Series (2000), 569. 
[11] BaBar, E. P. Solodov, SLAC, April 30 - May 2, 2001, hep-ex/0107027.

[12] Physics of the B factory book, Eur. Phys. J. C74 (2014) 3026.

[13] H. Nakazawa [Belle Collaboration], Nucl. Part. Phys. Proc. 260 (2015) 98.

[14] G. Pakhlova, Nucl. Phys. Proc. Suppl. 181-182 (2008) 117.

[15] M. Ablikim et al. [BESIII Collaboration], Phys. Lett. B 753 (2016) 629.

[16] A. Aloisio et al. [KLOE Collaboration], Phys. Lett. B 606 (2005) 12.

[17] G. W. Bennett et al. [Muon G-2 Collaboration], Phys. Rev. D 73 (2006) 072003.

For a review see J. P. Miller, E. de Rafael, B. Lee Roberts, Rep. Prog. Phys. 70 (2007) 795.

[18] S. Eidelman and F. Jegerlehner, Z. Phys. C 67 (1995) 585

[19] F. Teubert, talk at ICHEP04 Conference, http://www.ihep.ac.cn/data/ichep04/ppt/plenary/p21-teubert-f.ppt

[20] M. Davier, S. Eidelman, A. Hocker and Z. Zhang, Eur. Phys. J. C 27 (2003) 497.

[21] F. Ambrosino et al. [KLOE Collaboration], Phys. Lett. B 670 (2009) 285

[22] F. Ambrosino et al. [KLOE Collaboration], Phys. Lett. B 700 (2011) 102.

[23] H. Czyż, A. Grzelinska and J. H. Kühn, Phys. Lett. B 611 (2005) 116.

[24] F. Ambrosino et al. [KLOE Collaboration], Phys. Lett. B 634 (2006) 148.

[25] G. Pancheri, O. Shekhovtsova and G. Venanzoni, J. Exp. Theor. Phys. 106 (2008) 470;

G. Pancheri, O. Shekhovtsova and G. Venanzoni, Phys. Lett. B 642 (2006) 342; K. Melnikov,

F. Nguyen, B. Valeriani and G. Venanzoni, Phys. Lett. B 477 (2000) 114.

[26] R. R. Akhmetshin et al. [CMD-2 Coll.], Phys. Lett. B 648 (2007) 28.

[27] R. R. Akhmetshin et al. [CMD-2 Coll.], JETP Lett. 84 (2006) 413.

[28] M. N. Achasov et al. [SND Coll.], J. Exp. Theor. Phys. 103 (2006) 380.

[29] B. Aubert et al. [BABAR Collaboration], Phys. Rev. Lett. 103 (2009) 231801.

[30] D. Babusci et al. [KLOE Collaboration], Phys. Lett. B 720 (2013) 336.

[31] F. Jegerlehner and A. Nyffeler, Phys. Rept. 477 (2009) 1.

[32] M. Davier, A. Hoecker, B. Malaescu and Z. Zhang, Eur. Phys. J. C 71 (2011) 1515 Erratum:

[Eur. Phys. J. C 72 (2012) 1874].

[33] K. Hagiwara, R. Liao, A. D. Martin, D. Nomura and T. Teubner, J. Phys. G 38 (2011) 085003.

[34] F. Jegerlehner and R. Szafron, Eur. Phys. J. C 71 (2011) 1632.

[35] M. Benayoun, P. David, L. DelBuono and F. Jegerlehner, Eur. Phys. J. C 75 (2015) no.12, 613 doi:10.1140/epjc/s10052-015-3830-x.

[36] M. Davier, arXiv:1612.02743 [hep-ph].

[37] F. Jegerlehner, arXiv:1705.00263 [hep-ph].

[38] F. Curciarello, EPJ Web Conf. 118 (2016) 01008.

[39] A. Anastasi et al. [KLOE-2 Collaboration], Phys. Lett. B 767 (2017) 485.

[40] F. Jegerlehner, alphaQED package [version April 2012] http://www-com.physik.hu-berlin.de/ fjeger/alphaQED.tar.gz; see also F. Jegerlehner, Nuovo Cim. C 034S1 (2011) 31; Nucl. Phys. Proc. Suppl. 162 (2006) 22.

[41] F. Jegerlehner, EPJ Web Conf. 118 (2016) 01016.

[42] J. E. Augustin et al., Phys. Rev. Lett. 30 (1973) 462.

[43] J. Bailey et al. [CERN Muon Storage Ring Collaboration], Phys. Lett. 67B (1977) 225 [Phys. Lett. 68B (1977) 191].

[44] J. Bailey et al. [CERN-Mainz-Daresbury Collaboration], Nucl. Phys. B 150 (1979) 1.

[45] F. Jegerlehner, “The anomalous magnetic moment of the muon," Springer Tracts Mod. Phys. 226 (2008) 1 .

[46] K. A. Olive et al. [Particle Data Group], Chin. Phys. C 38 (2014) 090001. 
[47] M. Benayoun, EPJ Web Conf. 118 (2016) 01001; M. Benayoun, P. David, L. DelBuono and F. Jegerlehner, Eur. Phys. J. C 73 (2013) 2453; M. Benayoun, P. David, L. DelBuono and F. Jegerlehner, Eur. Phys. J. C 72 (2012) 1848; F. Jegerlehner and R. Szafron, Eur. Phys. J. C 71 (2011) 1632; M. Benayoun, P. David, L. DelBuono and O. Leitner, Eur. Phys. J. C 68 (2010) 355; M. Benayoun, P. David, L. DelBuono and O. Leitner, Eur. Phys. J. C 65 (2010) 211; M. Benayoun, P. David, L. DelBuono, O. Leitner and H. B. O’Connell, Eur. Phys. J. C 55 (2008) 199.

[48] J. Grange et al. [Muon g-2 Collaboration], arXiv:1501.06858 [physics.ins-det].

[49] N. Saito [J-PARC g-2/EDM Collaboration], AIP Conf. Proc. 1467 (2012) 45.

[50] C. M. Carloni Calame, M. Passera, L. Trentadue and G. Venanzoni, Phys. Lett. B 746 (2015) 325.

[51] G. Abbiendi et al., Eur. Phys. J. C 77 (2017) no.3, 139.

[52] L. Trentadue, these proceedings. 